\title{
Revisiting von Restorff's early isolation effect
}

\author{
Stephen R. Schmidt ${ }^{1}$ - Constance R. Schmidt ${ }^{1}$
}

Published online: 15 September 2016

(C) Psychonomic Society, Inc. 2016

\begin{abstract}
An item that is conceptually or physically different from other items in a series is often remembered well. This isolation effect has been found independent of the position of the isolated item in the list, suggesting that special attention to or processing of the isolated item is not a necessary precondition of the effect. Three experiments are reported that challenge this conclusion. In Experiment 1a, we compared memory for conceptually isolated items to memory for the same items in unrelated and homogeneous lists. Under moderately distracting conditions, isolation effects were observed with midlist but not with early isolates. In fact, early isolation impaired memory for the conceptually distinct items relative to the same items in homogeneous lists. Experiment $1 \mathrm{~b}$ replicated this memory impairment for early conceptual isolates and extended it to nondistracting conditions. In Experiment 2, we focused on early isolation, manipulating the type of isolation and whether or not participants performed judgments of learning (JOLs). An early isolation effect was observed for numbers isolated in lists of words (and vice versa), but not for conceptual isolates. Performing the JOL task reduced the size of the early isolation effect. These results suggest that number/word stimulus contrasts are coded automatically and support an isolation effect independent of list position. However, conceptual contrasts require relational processing and will only support an early isolation effect when such processing occurs. The results of Experiments 1a, 1b, and 2 suggest that attentional resources during list presentation and a favorable retrieval environment combine to support good memory for distinctive events.
\end{abstract}

Stephen R. Schmidt

Stephen.Schmidt@mtsu.edu

1 Psychology Department, Middle Tennessee State University, Box 87, Murfreesboro, TN 37132, USA
Keywords Von Restorff effect · Isolation effect · Recall · Distinctiveness

\section{Introduction}

The concept of distinctiveness is ubiquitous in discussions of human memory. It has been employed to explain a diverse set of findings, including flashbulb memories and all of the following effects: word frequency, primacy, recency, picture superiority, emotional memory, imagery, concreteness, and word length (see Schmidt, 1991, 2007, 2012, for reviews). In addition, distinctiveness has been successfully applied to areas outside the memory arena. For example, social psychologists have invoked distinctiveness to explain the formation of illusory correlations in the biased judgments of groups (McConnell, Sherman, \& Hamilton, 1994). Despite this widespread embrace of the concept, at least one empirical finding supporting theories of distinctiveness may not be as robust as has previously been assumed.

Von Restorff (1933) demonstrated that an item is remembered better when it "stands out" from a set of homogeneous items (e.g., a number in a list of nonsense syllables) than when it is embedded in a set of heterogeneous items (e.g., a color, a button, etc.). Good memory for an item that is conceptually or physically different from other items in a list is called the isolation effect. The isolation effect is the classic example of what Schmidt (1991, 2012) has called "primary distinctiveness," memory for events that differ physically, conceptually, or temporally from a background. Von Restorff's isolation effect occurred even when the isolated item appeared in the second or third position in the item series, a phenomenon called the early isolation effect. 
The early isolation effect is an important pillar supporting theories of distinctiveness. Von Restorff and others (e.g., Dunlosky, Hunt, \& Clark, 2000; Hunt, 1995, 2009) have rejected a variety of "extra processing" explanations for enhanced memory of distinctive events based on the early isolation effect. Green (1956) proposed one of these extra processing explanations, arguing that participants were surprised by the physical or conceptual changes that occurred when a distinctive item was presented in a series and that this surprise led to increased attention to and memory for the isolated item. Rundus (1971) also suggested that isolates received extra attention, leading to increased rehearsal and good memory for the distinctive items. Schmidt (1991) proposed that the incongruity between isolates and preceding items on the list increased storage of individual-item information important for good memory performance. However, neither surprise nor incongruity processing should occur when distinctive items appear early in the list, prior to the formation of expectations concerning list structure. Thus, the early isolation effect eliminates a whole class of explanations for the effects of distinctiveness on memory, including extra rehearsal or processing of the distinctive item. Many theorists have followed von Restorff's lead and endorsed discrimination and retrieval explanations of distinctiveness rather than attention or encoding explanations, concluding that distinctive items are less susceptible to interference or stand out in the memory representation relative to background items (e.g., Kelley \& Nairne, 2001; Waddill \& McDaniel, 1998). Others have argued that extra processing is not necessary but may contribute to good memory for distinctive events (e.g., Hunt, 1995).

The degree to which exclusion of extra processing explanations is warranted depends on the generality of the early isolation effect. If the early isolation effect occurs in only limited circumstances, then researchers may need to reconsider attentional resources in their explanations of primary distinctiveness. Evaluating the scope of the early isolation effect is particularly important given the broad inclusion of distinctiveness in explanations of memory and nonmemory phenomena. In the pages that follow, we present evidence for the role of attention in the midlist isolation effect and demonstrate that the early isolation effect occurs under limited conditions. We then present three experiments testing the boundary conditions of the early isolation effect. The results demonstrate that enhanced memory for unusual items is influenced by the amount and type of attentional resources deployed during encoding.

\section{Attention and the midlist isolation effect}

We will not attempt to review the extensive literature on the midlist isolation effect; several reviews can be found elsewhere (Wallace, 1965; Schmidt, 1991, 2008). Instead, we will focus on research demonstrating that shifts in resources during list processing play an important role in the midlist isolation effect.

Researchers have noted that increased skin conductance (Gati \& Ben-Shakhar, 1990) and the P300 cortical response (Fabiani \& Donchin, 1995; Fabiani, Karis, \& Donchin, 1990; Karis, Fabiani, \& Donchin, 1984) are associated with the isolation effect. These physiological measures indicate increased "encoding processes immediately following the presentation of distinctive events" (Fabiani \& Donchin, 1995, p. 237). Fabiani and Donchin (1995; see also Fabiani, 2006) argued that the P300 cortical response signals a "mismatch detection" that is associated with context updating and a reorganization of the list presentation experience. Following detection of the mismatch, additional processing may either support or erase the memory advantage typically associated with isolated items depending on the nature of that processing (see Fabiani, 2006).

Research with older adults also supports the conclusion that encoding processes are important to the isolation effect. Cimbalo and Brink (1982) failed to find an isolation effect in older adults when the isolated item was printed in a larger font than other items in the list. These researchers argued that the older adults ignored the change in font size, treating it as "analogous to noise" (p. 76). More recent studies have reported isolation effects in older adults, but the effects were smaller in magnitude than the effects found in college-age adults (Bireta, Surprenant, \& Neath, 2008; Geraci, McDaniel, Manzano, \& Roediger, 2009). Bireta et al. (2008) argued that older adults lack the cognitive resources needed for "processing the difference in the context of similarity" ( $p$. 351; see also Smith, 2011). Geraci et al. (2009) suggested that older adults lack the attentional control necessary for observing a strong isolation effect. Vitali et al. (2006) reported an isolation effect in older adults free from cognitive impairment that was not observed in a comparison group with Alzheimer's disease. These researchers suggested that the unimpaired participants responded to stimulus novelty with increased encoding and visual-perceptual processing; however, Alzheimer's participants failed to engage in extra processing of isolated items. Thus, the isolation effect is somewhat precarious in older populations either because older adults fail to notice some types of stimulus change or because they fail to process isolated items differently from other items in the list. Apparently, processing stimulus changes at encoding is an important component of the isolation effect.

The cognitive resources participants have available for encoding stimulus contrasts also contribute to the isolation effect. Schmidt and Schmidt (2015, Exp. 2) had students perform a Stroop color-naming task in preparation for a memory test. Half of the students performed a hard Stroop task requiring the identification of one of four font colors, whereas the other half performed a relatively easy two-color identification task. Three types of list structures were tested: isolation lists containing a target word from one category embedded in a list 
of words from a contrasting category (e.g., celery in a list of fish); homogeneous lists of words from a single category (e.g., vegetables), and lists of unrelated words. Correct color naming was slower for isolated target words than for targets embedded in homogeneous lists, suggesting that processing the isolated word required increased resources at encoding. In the easy Stroop task, conceptually isolated targets were better remembered than comparison targets in either the homogeneous or the unrelated lists. In the hard task, the isolation effect was only found when isolated targets were compared to targets in the unrelated lists. Schmidt and Schmidt argued that the hard Stroop task prevented extensive processing of list structure, thereby reducing the isolation effect. The isolation effect disappeared completely when the comparison condition was a homogeneous list of words from a single category. The limited encoding resources of the hard Stroop task combined with the retrieval advantages of recalling conceptually related items from the homogeneous list to eliminate the isolation effect. Thus, the isolation effect either decreases or disappears when cognitive resources are limited, depending on the nature and retrieval advantages of the comparison list.

Bireta and Mazzei (2015) also demonstrated that the isolation effect depends on having sufficient cognitive resources available to process stimulus changes within a list. Participants in their study recalled words that were either physically isolated (a red word embedded in a list of black words) or conceptually isolated (a word from one category presented in a list of words from a contrasting category). During list presentation, participants either counted backward or silently read the words. Physical isolates were recalled better than non-isolated targets in both presentation conditions. However, conceptual isolates were only recalled better than non-isolates in the silent reading condition. As we saw in the Schmidt and Schmidt (2015) study, the isolation effect is reduced or eliminated when cognitive resources for processing stimulus changes are limited. Further, cognitive resources during list presentation may play a particularly important role in governing the isolation effect when items are conceptually isolated.

\section{Review of the early isolation effect}

Von Restorff and others argued that the early isolation effect precludes increased processing of isolated items, yet we have just reviewed extensive literature demonstrating the importance of such processing in explaining the midlist isolation effect. With this conundrum in mind, we will now review the literature supporting the early isolation effect. A summary of early-isolation studies published in mainstream psychology journals appears in Table 1. The top section of the table includes studies demonstrating positive effects of early isolation on memory. With a few exceptions, each of these investigations demonstrated an isolation effect with the isolated item appearing in one of the first three positions in a list of homogeneous items. In the Kelley and Nairne (2001) study, the position of the isolate was systematically varied across all six serial positions in their lists. Geraci and Manzano (2010) placed the early isolates in one of the first four list positions. ${ }^{1}$ The lower section of the table lists studies that failed to show an early isolation effect or that reported an early isolation effect that was smaller in magnitude than a midlist isolation effect (e.g., Bone \& Goulet, 1968). Studies that failed to find either early or midlist isolation effects (e.g., Saltzman \& Carterette, 1959) and studies reporting ceiling effects that potentially masked the early isolation effect (e.g., Johansson, 1970) were excluded from the lower section of the table.

As can be seen in Table 1, von Restorff and numerous other researchers compared memory for isolated targets to memory for targets embedded in lists of unrelated items. In a notable exception to this rule, Hunt (2009) compared recall of an isolated word or number to recall of the same item in single-category lists of words or homogeneous lists of numbers, respectively. Schmidt and Schmidt (2015) argued that a word in a homogeneous control list has a retrieval advantage over the same word isolated in a contrasting category. Midlist isolates may require increased processing during presentation to overcome the retrieval advantage enjoyed by the homogeneous comparison lists. Schmidt and Schmidt (2015) demonstrated that the midlist isolation effect is larger and less susceptible to distraction when the comparison lists are unrelated words rather than words from a single category. Applying this logic to early isolation leads to the prediction that the early isolation effect may be less likely to occur with homogeneous than with unrelated control lists.

In a large majority of the successful demonstrations of the early isolation effect summarized in Table 1, digits were isolated in lists of verbal items (either consonant-vowel-consonant strings [CVCs] or words). In the unsuccessful attempts to demonstrate the effect reported in the table, items were isolated by font color (e.g., Huang \& Wille, 1979) or conceptual category (e.g., Elhalal, Davelaar, \& Usher, 2014). Thus, the early isolation effect may be limited to a restricted set of stimulus contrasts. Digits are both visually and conceptually different from verbal materials. Brain-imaging studies have shown that processing numbers and other ordered categories (e.g., months) engages a different part of the brain than processing conceptual categories (e.g., animals; see Ischebeck et al., 2008). Young children failed to show a midlist isolation effect with digits isolated in a series of pictures (Howe, Courage, Vernescu, \& Hunt, 2000). However, Smith (2011) demonstrated an early isolation effect with older adults when digits were isolated in a list of vehicle names (Exp. 2), but not

\footnotetext{
${ }^{1}$ In the often-cited Pillsbury and Raush (1943) experiment, the researchers systematically varied the number of isolates in the list. Although Pillsbury and Raush described their study as a replication of von Restorff's research, they did not specifically mention the serial position of the isolates.
} 
Table 1 Summary of experiments investigating early isolation effects

\begin{tabular}{|c|c|c|c|}
\hline Study & Type of Isolation & Control List & Task (Participants) ${ }^{*}$ \\
\hline \multicolumn{4}{|c|}{ Experiments demonstrating a positive early isolation effect } \\
\hline Bellezza \& Cheney (1973) & word underlined & unrelated words & $\begin{array}{l}\text { be sure to remember the } \\
\text { underlined word }\end{array}$ \\
\hline $\begin{array}{l}\text { Otani, Von Glahn, Libkuman, } \\
\text { Goernert, \& Kato (2014) }\end{array}$ & emotional & unrelated pictures & $\mathrm{JOL}^{* *}, \mathrm{IFR}^{* * *}$ \\
\hline Kelley \& Nairne (2001) & font size & unrelated words & order reconstruction \\
\hline von Restorff (1933, Exp. 3) & digits/syllables & unrelated items & IFR \\
\hline Pillsbury \& Raush (1943) & digits/syllables/words & none & IFR \\
\hline Postman \& Phillips (1954) & digits/syllables & none & IFR \\
\hline Hunt (1995) & digits/syllables & unrelated items & IFR \\
\hline Dunlosky et al. (2000, Exp. 1) & digits/syllables/words & unrelated items & JOL (Exp. 1), rehearsal (Exp. 2) \\
\hline Hunt (2009) & digits/words & all digits or words & IFR \\
\hline Geraci \& Manzano (2010, Exp. 2) & digits/syllables & unrelated symbols & delayed JOL, IFR \\
\hline Smith (2011, Exp. 2) & digits/words & digits & IFR (young \& old) \\
\hline Smith \& Hunt (2016, Exp. 1) & digits/trigrams & trigrams & delayed JOL, IFR (young \& old) \\
\hline Geraci \& Manzano (2010, Exp. 1) & category & unrelated words & delayed JOL, IFR \\
\hline Geraci \& Manzano (2010, Exp. 3) & category & unrelated words & category verification, IFR \\
\hline Smith (2011, Exp. 1) & category & unrelated words & IFR \\
\hline Smith \& Hunt (2016, Exp. 2) & category & unrelated words & delayed JOL, IFR \\
\hline \multicolumn{4}{|c|}{ Experiments in which the early isolation effect was absent or smaller in magnitude than the midlist isolation effect } \\
\hline Postman \& Phillips (1954) & digits/syllables & none & incidental free recall \\
\hline Bone \& Goulet (1968) & font color & CVCs & method of anticipation \\
\hline Huang, Scale, \& McIntyre (1976) & font color \& case & CVCs & IFR \\
\hline Huang \& Wille (1979) & font color & unrelated words & IFR \\
\hline Malhotra \& Dixit (1982) & font color & CVCs & delayed IFR \\
\hline Ludwig (2005, Exp. 1) & category & related words & JOL, IFR \\
\hline Elhalal, Davelaar, \& Usher (2014) & category & related \& unrelated words & IFR \\
\hline Smith (2011, Exp. 1) & category & unrelated words & IFR (old adults) \\
\hline Smith \& Hunt (2016, Exp. 2) & category & unrelated words & delayed JOL, IFR (old adults) \\
\hline
\end{tabular}

when the word table was isolated in a list of fish (Exp. 1). Perhaps adults code the contrast between digits and verbal materials relatively automatically, whereas young children do not. Observing the contrast between conceptual categories may require attention-demanding relational processing. When such processing is prevented by a difficult and distracting Stroop task (Schmidt \& Schmidt, 2015), by the limited cognitive resources that accompany aging (Smith, 2011; Smith \& Hunt, 2016), or by isolating items early in a list before the list structure is apparent, the conceptual isolation effect may disappear.

In fact, only four experiments have reported an early isolation effect with items isolated by conceptual category: Smith (2011, Exp. 1), Geraci and Manzano (2010, Exps. 1 and 3), and Smith and Hunt (2016, Exp. 2). Smith (2011) reported that young adults remembered words isolated by category better than the same words embedded in lists of unrelated words. This isolation effect was observed regardless of whether the targets were isolated early (Position 2) or in the middle (Position 5) of an eight-word list. Geraci and Manzano (2010, Exp. 1) investigated whether items isolated early in a list gained salience as the list structure became apparent. Others have suggested that early isolates are not salient because participants have not yet discovered the list structure when the isolate is presented (e.g., Dunlosky et al., 2000). Geraci and Manzano argued that, "salience arises over the course of the encoding session, once the study context has been revealed" (p. 52). To test this assertion, Geraci and Manzano asked participants to perform delayed judgments of learning (JOLs). Their word lists contained target words isolated by category or targets in a comparison list of unrelated words. The isolates appeared either early (Positions 1-4) or late (Positions 9-12) in the 14-word lists. The participants saw the target, saw two intervening items, and then saw the target a 
second time and were asked how likely they were to remember it. Isolated targets were given higher JOLs and were better recalled than control targets, independent of list position.

Smith and Hunt (2016) also demonstrated early isolation effects with delayed JOL tasks, although conceptually isolated items did not receive higher JOLs than control items in their second experiment. Geraci and Manzano (2010, Exp. 3) found an early isolation effect with conceptual isolates when participants were asked verify the category of the isolate immediately after it was presented. The delayed JOL and immediate category verification tasks may have encouraged relational processing of list items, increasing the likelihood of an early isolation effect. Note also that the control lists in all four studies demonstrating an early isolation effect with conceptual isolates (Geraci \& Manzano, 2010, Exps. 1 and 3; Smith, 2011; Smith \& Hunt, 2016) contained unrelated words, potentially limiting the generalizability of the effect.

A study by Soderstrom, Clark, Halamish, and Bjork (2015) supports our suggestion that JOLs influence how participants process a list of items. In a paired-associate learning task, the Soderstrom et al. participants focused on the associative strength of the word pairs while making JOLs. As a result, JOLs enhanced memory for strongly related pairs without affecting memory for unrelated pairs. Performing JOLs during a conceptual isolation task might similarly encourage processing of category relations among list items, thereby enhancing the early isolation effect. Participants making delayed JOLs see multiple items from the background category before evaluating the isolated item, further increasing the probability that they will process the relevant conceptual dimension (see Smith \& Hunt, 2016).

\section{Present studies}

Several of the demonstrations of early isolation effects may be attributed to factors other than isolation per se, including item priority, arousal, and the use of JOL or category verification tasks that potentially emphasize list structure. ${ }^{2}$ The review above also highlighted several variables that might limit the

\footnotetext{
${ }^{2}$ At least two of the experiments described in Table 1 do not provide definitive evidence for an early isolation effect. Bellezza and Cheney (1973) explicitly told their participants, "that whenever an underlined word appeared he should try to make sure that he would be able to recall it" (p. 56). Thus, this experiment demonstrated the effect of item priority (see, e.g., Tulving, 1969) rather than an isolation effect. Otani, Von Glahn, Libkuman, Goernert, and Kato (2014) isolated an arousing negative picture in a list of neutral pictures. Schmidt and his colleagues (Schmidt, 2006, 2007; Schmidt \& Saari, 2007; Schmidt \& Schmidt, 2015) have demonstrated that the effects of embedding emotional items in lists of neutral items are functionally different from typical isolation effects. The Otani et al. study provided additional evidence that emotional contrasts lead to atypical isolation effects, by demonstrating that a neutral picture isolated in a list of negative pictures was remembered poorly.
}

generality of the early isolation effect, including the type of memory test, the nature of the comparison list, and how target items were isolated. The experiments presented below were designed to determine which of these variables is critical for observing the early isolation effect. We assumed that the number of lists presented is not a critical variable, because it has varied from one list of items (e.g., Dunlosky et al., 2000) to as many as 54 lists (Kelley \& Nairne, 2001) in successful demonstrations of early isolation. To maximize statistical power, our experiments included multiple study-test sequences. Similarly, the timing of memory tests does not appear to be a critical variable in early isolation studies; the effect has been detected with tests immediately following list presentation (e.g., Bellezza \& Cheney, 1973; Kelley \& Nairne, 2001), and with tests delayed by as much as $48 \mathrm{~h}$ (e.g., Hunt, 2009). We employed immediate memory tests to maximize the number of lists presented. Free recall of our lists was also intentional, consistent with most of the experiments in Table 1.

On the basis of our review, we hypothesized that the nature of the control list, the type of isolation, the attentional resources available during list presentation, and whether or not participants make JOLs during list presentation might be determining factors in demonstrating the early isolation effect. In Experiment 1a, we explored the limiting conditions of conceptual isolation by comparing early and late isolation effects using different types of control lists under mildly distracting presentation conditions. Experiment $1 \mathrm{~b}$ replicated the early isolation conditions of Experiment 1a and assessed the impact of distraction during presentation on the early isolation effect. In Experiment 2, we contrasted isolation by conceptual category with isolation of a number in a list of words (and vice versa), and investigated the role of JOLs in the early isolation effect.

\section{Experiment 1a}

The primary purpose of Experiment 1a was to extend the logic and methods employed in Schmidt and Schmidt (2015) to the early isolation effect. Participants performed an easy colornaming Stroop task while preparing for immediate recall tests. They were asked to remember three types of lists: isolation lists containing a target item from one category embedded in a contrasting category, homogeneous comparison lists of items from a single conceptual category, and comparison lists of unrelated words. Target words appeared in either the second (early) or eighth (midlist) position of the 15-word lists. We expected to find the typical isolation effect for target words appearing midlist, replicating Schmidt and Schmidt (2015). However, we were uncertain whether the early isolation effect would be observed. Based on the review presented above (see Table 1), we thought that the early isolation effect might be found with comparison lists of unrelated words but not with homogeneous comparison lists. Alternatively, the color- 
naming task might prevent processing of list structure and interfere with an early isolation effect. Conceptual isolation might also be less likely to produce an early isolation effect than stimulus contrasts between numbers and verbal materials. Finally, we did not expect to observe an early isolation effect in Experiment 1 if the effect depends on performance of JOLs that orient participants toward list structure. If one or more of these variables limit observation of the early isolation effect, we should find a midlist but not an early isolation effect in Experiment 1a.

\section{Method}

Participants On the basis of previous research (Schmidt \& Schmidt, 2015), our goal was to obtain data from 80 participants, ten in each of the eight counterbalancing conditions (see below). Eighty-seven students from the Psychology Department's research pool were tested. Data from one student were omitted because the student failed to complete the color-naming task; the data from two additional students were excluded due to computer malfunctions. The final data set included between nine and 11 participants in each counterbalancing cell.

Materials The materials were the same as those employed in Schmidt and Schmidt (2015, Exp. 2). One practice list of 15 unrelated words was constructed from the Janschewitz (2008) word norms. Six experimental lists included: two isolation lists with a target item from one category embedded in a list of items from a contrasting category, two homogeneous comparison lists of items from a single category, and two unrelated comparison lists containing targets from one of the categories embedded in a list of unrelated words. For the experimental lists, 15 category members were selected from each of eight Van Overschelde, Rawson, and Dunlosky (2004) categories. Words listed in the eighth or ninth category positions were designated as target items. The target word appeared in the second list position for half of the list types and the eighth list position for the remaining half of the lists. Background words in the unrelated lists were selected from the Janschewitz (2008) norms. Target and background words were counterbalanced across participants and list types by rotating each category through eight list assignments. The items from each category were used as (1) the target in an early isolation list, (2) the target in a midlist isolation list, (3) the contrasting background items in an early isolation list, (4) the contrasting background items in a midlist isolation list, (5) the target in an early homogeneous comparison list, (6) the target in a midlist homogeneous comparison list, (7) the target in an early unrelated comparison list, and (8) the target in a midlist unrelated comparison list. None of the participants saw the same item or category more than once. Thus, a participant might see celery in a list of vegetables, tuna in a list of clothing, and violin in a list of unrelated words. A different participant would see tuna in a list of fish, violin in a list of vegetables, and celery in an unrelated comparison list.

Procedure The experimental task was controlled by E-Prime 2.0 software running on standard Windows computers with standard keyboards and 15-in. CRT monitors. Participants were tested in groups of one to six in a small laboratory space. The session began with an experimenter reading instructions aloud as they appeared on the computer screen. The participants then completed 40 practice color-naming trials in which the symbols “\#\#\#\#\#\#" appeared centered on the screen. The participants were instructed to type " $r$ " when the symbols appeared in red font and " $b$ " when the symbols appeared in blue font. The participants were asked to respond as quickly as possible, and were given feedback concerning the accuracy of their responses. Following this practice, the participants were given instructions concerning presentation of the word lists. Words were presented one at a time on the computer screen in either red or blue font. The words disappeared after the participants made a color-naming response, and were followed by a 1-s interword interval and the next word in the series. Seven study-test sequences (one practice plus six experimental lists) were completed. In the initial instructions, and prior to each list, participants were asked to respond to the word color as quickly as possible, and to study the list in preparation for the immediate memory test. Each participant studied two lists of each list type (isolation, homogeneous comparison, unrelated comparison), one with a target word in position two and the other with a target in Position 8 of the 15 -item list. Thus, the experimental design was a 3 (List Type: isolation, homogeneous comparison, unrelated comparison) $\times 2$ (Target Position: early vs. midlist) within-participants design. With the exclusion of the practice list that always came first, list order was randomly determined for each participant. Each list was immediately followed by a 60 -s recall period. Participants were instructed to type their responses on the computer.

\section{Results and discussion}

The results were analyzed in three separate 3 (List Type: isolation, homogeneous comparison, unrelated comparison) $\times 2$ (Target Position: early, midlist) $\times 2$ (Item: target, background) repeated measures analyses of variance (ANOVAs). The dependent measures for the three ANOVAs were color-naming accuracy, color-naming correct response times, and proportions of correct recall, respectively. A $p<.05$ was adopted for all statistical tests.

Color naming Color-naming accuracy was high $(M=.96)$ and relatively invariant across conditions. Only the interaction between item and position approached significance in the 
accuracy analysis, $F(1,166)=3.76, M S E=.019, p=.056, \eta_{\mathrm{p}}{ }^{2}$ $=.04$. Accuracy for early targets was lower $(M=.94)$ than accuracy for midlist targets $(M=.97)$. However, color-naming accuracy for background items did not vary as a function of target position (early position $M=.96$, midlist position $M=$ $.95)$. List type was the only significant effect in the colornaming response time analysis, $F(2,126)=4.92, M S E=$ 44644, $\eta_{\mathrm{p}}{ }^{2}=.072$. Responses to isolation lists $(M=808 \mathrm{~ms})$ were slower than responses to homogeneous $(M=779 \mathrm{~ms})$ and unrelated $(M=749 \mathrm{~ms})$ comparison lists. The two colornaming analyses suggested that participants were sensitive to list structure during list presentation, and that list structure had a larger impact when the isolated targets appeared midlist than when they appeared in list Position 2.

Recall A summary of the recall results can be found in Table 2. The effect of list type was significant, $F(2,166)=$ $32.69, M S E=.109, \eta_{\mathrm{p}}^{2}=.283$, as were the interactions between list type and target position, $F(2,166)=4.41, M S E=$ $.115, \eta_{\mathrm{p}}{ }^{2}=.050$, and item and target position, $F(2,166)=$ 4.83, MSE $=.148, \eta_{\mathrm{p}}{ }^{2}=.055$, and the three-way interaction between list type, item, and target position, $F(2,166)=6.14$, $M S E=.115, \eta_{\mathrm{p}}{ }^{2}=.069$. As can been seen in Table 2, the traditional isolation effect occurred with midlist but not with early isolates. Isolated targets in Position $8(M=.55)$ were recalled better than Position 8 targets from homogeneous comparison lists $(M=.43), t(83)=1.56, p=.06$, and unrelated comparison lists $(M=.27), t(83)=4.23$. In the midlist isolation conditions, background items from contrasting categories ( $M=.53)$ were recalled more poorly than the same background items in the homogeneous comparison lists $(M=$ $.57), t(83)=2.15$. In contrast, targets isolated in the early position $(M=.44)$ were recalled more poorly than targets in homogeneous comparison lists $(M=.63), t(83)=2.69$, and no better than targets in unrelated comparison lists $(M=.44)$, $t(83)=0.00$. In the early isolation conditions, no differences

Table 2 Target and background item memory as a function of list structure and position of the target in Experiment 1a

\begin{tabular}{lll}
\hline & \multicolumn{2}{l}{ Item } \\
\cline { 2 - 3 } List Type & Target Recall & Background Recall \\
\hline Early Isolation (Position 2) & & \\
$\quad$ Isolation & $.44(.05)^{*}$ & $.53(.02)$ \\
$\quad$ Homogeneous comparison & $.63(.05)$ & $.54(.02)$ \\
$\quad$ Unrelated comparison & $.44(.05)$ & $.33(.02)$ \\
Late Isolation (Position 8) & & \\
$\quad$ Isolation & $.55(.06)$ & $.53(.02)$ \\
$\quad$ Homogeneous comparison & $.43(.05)$ & $.57(.02)$ \\
$\quad$ Unrelated comparison & $.27(.05)$ & $.36(.01)$ \\
\hline
\end{tabular}

* Parenthetical values are standard errors of the means were observed between recall of the same background items in the isolation $(M=.53)$ and homogeneous $(M=.54)$ comparison lists, $t(83)=0.50$. Apparently, early isolation caused the targets to "sink into" rather than "stand out from" a homogeneous background category, a phenomenon similar to inattentional blindness (see Schmidt \& Schmidt, 2015). Elhalal et al. (2014) reported a similar pattern of results when they presented conceptual isolates in the first position of 12item lists.

The most striking finding in Experiment 1a was the absence of an early isolation effect under the same conditions and with the same participants as a midlist isolation effect. The early isolation effect may therefore occur under more limited conditions than the midlist effect. Which task components of Experiment 1a constrained observation of the early isolation effect? Perhaps the attentional demands of the color-naming task disrupted the early more than the late isolation effect. As a test of this hypothesis, in Experiment $1 \mathrm{~b}$ we compared the early isolation effects across two groups of participants; one group simply read lists of words, and a second group performed the easy color-naming task employed in Experiment 1a.

\section{Experiment 1b}

\section{Method}

Participants The participants in Experiment $1 \mathrm{~b}$ were $101 \mathrm{stu}-$ dents from the same source as Experiment 1a. The data from two participants were discarded because of computer malfunctions. Three additional participants were randomly excluded so that the read and color-naming conditions each included 48 participants.

Materials The materials were identical to those employed in Experiment 1a.

Procedure As in Experiment 1a, each participant studied and was tested on seven lists of items in an intentional free recall task. For participants in the read condition, each word was presented in black font for $2 \mathrm{~s}$ followed by a 1-s interstimulus interval (ISI). With one exception, the procedure for the colornaming condition was identical to the color-naming procedure in Experiment 1a. The exception was that the stimulus first appeared in black font for $2 \mathrm{~s}$ and then changed to red or blue font; this revised procedure insured that stimulus duration was approximately the same in the color naming and read conditions. The participants' color-naming response then terminated the stimulus. The revised color-naming task was no longer sensitive to initial differences in response latencies between isolated and nonisolated items; thus, response time data are not reported in Experiment 1b. 


\section{Results and discussion}

The recall results were analyzed with a 2 (Task: read, color naming) $\times 3$ (List Type: isolation, homogeneous comparison, unrelated comparison) $\times 2$ (Item Type: target, background) mixed ANOVA. Task was a between-participants factor, whereas List Type and Item Type were within-participants factors. A summary of the recall results can be found in Table 3.

Memory in the read condition $(M=.63)$ exceeded memory in the color-naming condition $(M=.55), F(1,94)=8.42, M S E$ $=.098, \eta_{\mathrm{p}}{ }^{2}=.082$. The effect of task did not interact with either list type, $F(2,188)=1.99, M S E=.049, \eta_{\mathrm{p}}{ }^{2}=.021$, or item type, $F(1,94)=1.63, M S E=.063, \eta_{\mathrm{p}}{ }^{2}=.017$. List type significantly influenced memory performance, $F(2,188)=$ 20.06, MSE $=.049, \eta_{\mathrm{p}}{ }^{2}=.176$, as did item type, $F(1,94)=$ $55.74, M S E=.063, \eta_{\mathrm{p}}{ }^{2}=.372$. These effects were compromised by a significant interaction between list type and item type, $F(2,188)=12.87, M S E=.054, \eta_{\mathrm{p}}{ }^{2}=.120$.

Most importantly, as can be seen in Table 3, there was no evidence for an early isolation effect in either the read or the color-naming condition. Quite the contrary, targets isolated by category were recalled more poorly than targets in the homogeneous comparison lists in both the read condition (isolation $M=.68$, homogeneous comparison $M=.82), t(47)=2.36$, and the color-naming condition (isolation $M=.52$, homogeneous comparison $M=.69), t(47)=2.77$. In the read condition, recall of isolated targets $(M=.68)$ was almost identical to recall of targets in the unrelated list $(M=.66), t(47)=0.47$. In the colornaming task, recall of isolated targets $(M=.52)$ was marginally poorer than recall of targets in the unrelated list $(M=.65)$, $t(47)=1.90$, two-tailed $p=.06 .^{3}$

Under distracting conditions in Experiments $1 \mathrm{a}$ and $1 \mathrm{~b}$, early isolation hurt memory for the isolated items in a homogeneous control list, and had no effect using an unrelated control list. This pattern of results was extended to nondistracting list presentations in Experiment $1 \mathrm{~b}$. The absence of early isolation effects in both experiments suggests that the type of isolation, the use of JOL tasks during list presentation, or both of these task components might play important roles in the early isolation effect.

\footnotetext{
${ }_{3}^{3}$ Several researchers have expressed concern that multiple study-test sequences may alert participants to the manipulation of list structure, and thereby change the isolation effect (e.g., Schmidt, 2007; Smith, 2011). To address this concern, we conducted a second analysis of the results that included only the data from the first experimental list. This created a between-participants design, rather than a within-participants design, sacrificing statistical power. Nonetheless, the pattern of the results (combining the read and color-naming conditions) was unchanged. Recall of targets from homogeneous lists $(M=.84)$ was greater than recall of targets from isolated lists $(M=.73), t(59)=1.00, p=.32$, and targets from unrelated lists were also recalled better $(M=.79)$ than isolated targets, $t(63)=0.49$. However, none of these planned comparisons was significant.
}

Table 3 Target and background item memory as a function of list structure and task in Experiment $1 \mathrm{~b}$

\begin{tabular}{lll}
\hline & \multicolumn{2}{l}{ Item } \\
\cline { 2 - 3 } List Type & Target Recall & Background Recall \\
\hline Read & & \\
$\quad$ Isolation & $.68(.05)^{*}$ & $.61(.02)$ \\
Homogeneous comparison & $.82(.05)$ & $.59(.02)$ \\
Unrelated comparison & $.66(.05)$ & $.41(.02)$ \\
Color Naming & & \\
$\quad$ Isolation & $.52(.05)$ & $.55(.02)$ \\
Homogeneous comparison & $.69(.05)$ & $.55(.02)$ \\
Unrelated comparison & $.65(.05)$ & $.37(.02)$ \\
\hline
\end{tabular}

"Parenthetical values are standard errors of the means

\section{Experiment 2}

The literature review summarized in Table 1 led us to suspect that the early isolation effect is more robust when numbers are isolated in verbal materials than with the conceptual-category contrasts examined in Experiments $1 \mathrm{a}$ and 1b. Perhaps adults automatically detect the difference between numbers and words, and this difference is represented in memory even when the isolated item is presented early in a series. In contrast, storing the conceptual difference between a fish and musical instruments may require relational processing, which may occur under limited circumstances. In Experiment 2, we explored the effects of type of isolation on the early isolation effect by comparing memory for conceptual isolates with memory for words isolated in numbers and numbers isolated in words. We predicted a robust early isolation effect for isolation lists involving word/number contrasts. As a strong test of this robustness, we compared isolated targets to targets in homogeneous control lists. As we have shown repeatedly (e.g., Schmidt \& Schmidt, 2015, and Exps. 1a and 1b above), the isolation effect is more difficult to obtain with homogeneous than with unrelated control lists.

A second issue addressed in Experiment 2 was the role of JOLs in supporting the early isolation effect. As we noted above, asking participants to perform a JOL task may encourage relational processing across the list. The JOL task may prompt participant elaboration of the differences between the isolated item and other items in the list, increasing the likelihood of observing an early isolation effect. Alternatively, the JOLs may enhance individual-item processing as participants use intrinsic cues to judge whether or not each item is memorable (Koriat, 1997). The JOL task may be functionally similar to the "difference judgment" task employed by Hunt and Lamb (2001), increasing individual-item processing at the expense of relational processing across list items. Hunt and Lamb found that difference judgments reduced the traditional 
midlist isolation effect. Thus, JOLs could potentially reduce rather than enhance the early isolation effect. In Experiment 2, participants were given either read or JOL instructions to determine the impact of JOLs on the early isolation effect.

\section{Method}

Participants One hundred and sixteen participants were recruited from the psychology research pool and randomly assigned to two tasks conditions (read vs. JOL). One participant was excluded for failing to follow instructions; the data from four additional participants were discarded due to computer malfunctions. The final analysis included 58 participants in the read condition and 53 participants in the JOL condition.

Materials Six categories were selected from the Van Overschelde et al. (2004) category norms: clothing, fish, countries, occupations, musical instruments, and elements. Fifteen items were selected from these categories, and one item was selected as the target item in the same manner as in Experiments $1 \mathrm{a}$ and $1 \mathrm{~b}$. In addition, 45 random two-digit numbers were selected from the numbers between 10 and 99 . Three of these numbers were randomly assigned as the targets $(39,54$, and 25). From these materials we created three types of isolation lists: lists of words from one category containing a target word from a contrasting conceptual category, lists of numbers containing a single target word, and lists of words from a single category containing one target number. Additionally, three homogeneous comparison lists were created; two were homogeneous lists of words from a single category, and the third was a homogeneous list of numbers. In a procedure similar to that employed in Experiments $1 \mathrm{a}$ and $1 \mathrm{~b}$, words and numbers were counterbalanced across participants so that each target word or number appeared in each applicable list structure. In this manner, we created a 3 (Type of Isolation: words in words, words in numbers, numbers in words) $\times 2$ (List Type: isolation, homogeneous comparison) within-participants design. For example, a participant might see the following lists (in random order): 39 in a list of clothing, tuna in a list of fish, 25 in a list of numbers, zinc in a list of numbers, Italy in a list of occupations, and violin in a list of musical instruments. An additional eight unrelated words and eight two-digit numbers served as an item pool for a practice list. Fifteen of these items were selected at random for each participant for the practice list that preceded the six experimental lists.

Procedure Each participant studied seven lists of 15 items in preparation for immediate recall tests. In the read condition, each item was presented for $2 \mathrm{~s}$, followed by a 1-s ISI. In the
JOL condition, each item was presented, and participants were immediately asked, "How confident are you that at the end of the list you will be able to recall the item?"4 Following Dunlosky et al. (2000), the participants were asked to rate their confidence on a scale from 0 (definitely will not recall) to 100 (definitely will recall). The JOL task terminated word presentation, and 1-s blank screens separated the words. The participants in both conditions were given $1 \mathrm{~min}$ to type their recall immediately following each list.

\section{Results and discussion}

JOL ratings JOL response times averaged $4.33 \mathrm{~s}(S D=1.93)$. The JOL ratings were analyzed to determine whether the isolated items were salient during presentation. Ratings of the target items were analyzed with a 3 (Type of Isolation: words in words, words in numbers, numbers in words) $\times 2$ (List Type: isolation, homogeneous comparison) withinparticipants ANOVA. Only the effects of type of isolation, $F(2,98)=13.65, M S E=512.072, \eta_{\mathrm{p}}{ }^{2}=.218$, and list type, $F(1,49)=6.77, M S E=389.036, \eta_{\mathrm{p}}{ }^{2}=.121$, were significant. The interaction between these two factors did not approach significance, $F(2,98)=0.63, M S E=404.218, \eta_{\mathrm{p}}{ }^{2}=.013$. Both target words presented in lists of words $(M=58.53)$ and target words embedded in lists of numbers $(M=58.37)$ were rated as easier to learn than target numbers presented in lists of words $(M=43.97), t \mathrm{~s}(49)=4.25$ and 4.20 , respectively. Additionally, isolated items were rated as harder to learn $(M$ $=50.66)$ than items in homogeneous lists $(M=56.59), t(49)=$ 2.06. Thus, participants appeared to notice that the isolated items were different, but they did not expect to be able to remember them better than other list items.

Recall Free recall was analyzed with a 3 (Type of Isolation: words in words, words in numbers, numbers in words) $\times 2$ (List Type: isolation, homogeneous comparison) $\times 2$ (Type of Item: target, background) $\times 2$ (Task: read, JOL) mixed ANOVA. Task was a between-participants factor; the other factors were manipulated within participants. A summary of the means can be found in Tables 4 and 5. Main effects of type of isolation, $F(2,218)=36.61, M S E=.093, \eta_{\mathrm{p}}{ }^{2}=.251$, and type of item, $F(1,109)=73.72, M S E=.129, \eta_{\mathrm{p}}{ }^{2}=.40$, were observed. The main effect of task was not significant, $F(1$, 109) $=0.52, M S E=.21, \eta_{\mathrm{p}}{ }^{2}=.005$. However, interactions were observed between type of isolation and task, $F(1,109)=$ 4.66, MSE $=.089, \eta_{\mathrm{p}}{ }^{2}=.041$, and type of item and task, $F(1$,

\footnotetext{
${ }^{4}$ Consistent with the Dunlosky et al. (2000, Exp. 1) study, we used immediate rather than delayed JOLs in Experiment 2. Our reasoning was that delayed JOLs would contaminate the early-isolation manipulation by requiring a second presentation of the isolate following two intervening background items. This second presentation would push the isolate later in the presentation list, thereby providing an alternative interpretation of any observed isolation effect.
} 
Table 4 Target and background item memory as a function of type of isolation, list structure, and task in Read condition of Experiment 2

\begin{tabular}{lll}
\hline & \multicolumn{1}{l}{ Item } & \\
\cline { 2 - 3 } $\begin{array}{l}\text { Type of Isolation/ } \\
\text { List Type }\end{array}$ & $\begin{array}{l}\text { Target } \\
\text { Recall }\end{array}$ & $\begin{array}{l}\text { Background } \\
\text { Recall }\end{array}$ \\
\hline Conceptual Category & & \\
Isolation & $.71(.06)^{*}$ & $.60(.02)$ \\
$\begin{array}{l}\text { Homogeneous } \\
\text { comparison }\end{array}$ & $.84(.05)$ & $.62(.02)$ \\
$\begin{array}{l}\text { Word in Numbers } \\
\text { Isolation }\end{array}$ & $.90(.05)$ & $.33(.02)$ \\
$\begin{array}{l}\text { Homogeneous } \\
\text { comparison } \\
\text { (all words) }\end{array}$ & $.79(.05)$ & $.59(.02)$ \\
$\begin{array}{l}\text { Number in Words } \\
\text { Isolation }\end{array}$ & $.72(.06)$ & \\
$\begin{array}{l}\text { Homogeneous } \\
\text { comparison } \\
\text { (all numbers) }\end{array}$ & $.45(.07)$ & $.61(.03)$ \\
\hline
\end{tabular}

* Parenthetical values are standard errors of the means

109) $=7.12, M S E=.129, \eta_{\mathrm{p}}{ }^{2}=.061$. The three-way type of list by type of item by task interaction was also significant, $F(1,109)=4.54, M S E=.081, \eta_{\mathrm{p}}^{2}=.040$. Additional interactions included type of isolation by list type, $F(2,218)=36.11$, $M S E=.086, \eta_{\mathrm{p}}{ }^{2}=.249$, type of isolation by type of item, $F(2$, $218)=34.04, M S E=.094, \eta_{\mathrm{p}}{ }^{2}=.238$, and the three-way interaction involving type of isolation, list type, and type of

Table 5 Target and background item memory as a function of type of isolation, list structure, and task in JOL condition of Experiment 2

\begin{tabular}{lll}
\hline & \multicolumn{1}{l}{ Item } & \\
\cline { 2 - 3 } $\begin{array}{l}\text { Type of } \\
\text { Isolation/ }\end{array}$ & $\begin{array}{l}\text { Target } \\
\text { List Type }\end{array}$ & $\begin{array}{l}\text { Background } \\
\text { Recall }\end{array}$ \\
\hline $\begin{array}{l}\text { Conceptual Category } \\
\text { Isolation }\end{array}$ & & \\
& & $.68(.02)$ \\
Homogeneous & $(.06)^{*}$ & $.67(.02)$ \\
comparison & $.81(.05)$ & \\
Word in Numbers & & $.40(.02)$ \\
$\begin{array}{l}\text { Isolation } \\
\text { Homogeneous } \\
\text { comparison } \\
\text { (all words) }\end{array}$ & $.83(.05)$ & $.64(.02)$ \\
$\begin{array}{l}\text { Number in Words } \\
\text { Isolation }\end{array}$ & $.87(.05)$ & \\
$\begin{array}{l}\text { Homogeneous } \\
\text { comparison } \\
\text { (all numbers) }\end{array}$ & & $.69(.03)$ \\
\hline
\end{tabular}

* Parenthetical values are standard errors of the means item, $F(2,218)=15.19, M S E=.097, \eta_{\mathrm{p}}{ }^{2}=.122$. Because we were primarily interested in recall of the targets, and in light of the multiple interactions involving type of item, the ANOVA was repeated with only the target items. Once again, we found a main effect of list type, $F(2,218)=31.15, M S E=.169, \eta_{\mathrm{p}}{ }^{2}=$ .222 , and interactions between list type and task, $F(1,109)=$ $5.00, M S E=.156, \eta_{\mathrm{p}}{ }^{2}=.044$, and type of isolation and list type, $F(2,218)=6.37, M S E=.165, \eta_{\mathrm{p}}^{2}=.055$. We conducted cell-by-cell tests of the isolation effect to clarify these interactions. ${ }^{5}$

In the read condition, recall of target words isolated by conceptual category $(M=.71)$ was worse than recall of the same target words in the homogeneous lists $(M=.84), t(57)=$ 1.74. This finding replicates the first two experiments, demonstrating that early isolation hurts recall of the isolate relative to the same item in a homogeneous comparison list. In contrast, a word isolated in a list of numbers $(M=.90)$ was recalled better than a word embedded in a homogeneous comparison list of words from a single category $(M=.79), t(57)=$ 1.76. Similarly, a number isolated in a list of words $(M=.72)$ was recalled better than a number embedded in a homogeneous list of numbers $(M=.45), t(57)=3.41$. Thus, the early isolation effect was confined to contrasts between numbers and words, a finding consistent with much of the research presented in Table 1. Numbers and words are both conceptually and visually dissimilar, differences that may automatically be coded in memory even early in a list of items, before the list structure becomes apparent.

Recall following the JOL task was quite different from recall following the read task. Only one $t$ test approached significance: Words isolated by conceptual category $(M=$ .68) were recalled marginally more poorly than the same words embedded in homogeneous, single-category lists $(M=$ $.81), t(57)=1.63$, one-tailed $p=.055$. These results suggest that the JOL task increases individual-item processing rather than relational processing across the list. The JOL results of Experiment 2 resemble the results of Hunt and Lamb (2001), in that the isolation effect is diminished when participants focus on individual items. At first glance, our memory results following the JOL task may appear inconsistent with the earlier reports of the isolation effect summarized in Table 1. However, in all of the earlier studies employing the JOL task, the comparison lists contained unrelated items. Experiments $1 \mathrm{a}$ and $1 \mathrm{~b}$ reported above demonstrated that recall of targets in

\footnotetext{
$\overline{5}$ One should be cautious comparing the specific cells in Tables 4 and 5, because some comparisons are between memory for words and memory for numbers. Words were clearly easier to remember than numbers. For example, recall of background words in the all-words comparison list for the words-in-numbers isolation list $(M=.61, S E M=.015)$ was greater than recall of background numbers in the all-numbers comparison list for the numbers-in-words isolation list $(M=.39, S E M=.014)$. The most interesting and appropriate comparisons contrast recall of the same item embedded in different background lists. Only these comparisons were tested with $t$ tests and are reported here.
} 
comparison lists is greatly enhanced when these lists contain related rather than unrelated items. In fact, recall of targets from homogeneous comparison lists in Experiment 1a was as much as .19 higher than recall of targets from unrelated comparison lists (see Table 2). Had we employed unrelated control lists in Experiment 2, we probably would have observed an early isolation effect with mixtures of words and numbers following the JOL task.

Recall of background items further elucidated the effect of task on overall memory performance. Recall of background items in the JOL condition $(M=.59)$ was greater than recall of background items in the read condition $(M=.51), F(1,109)=$ $14.26, M S E=.058, \eta_{\mathrm{p}}{ }^{2}=.116$. Furthermore, the effect of task on background memory was not compromised by interactions between task and type of isolation, $F(2,218)=1.77, M S E=$ $.018, \eta_{\mathrm{p}}{ }^{2}=.016$, or task and list type, $F(1,109)=0.05, M S E=$ $.014, \eta_{\mathrm{p}}^{2}=.000$. One interpretation of these findings is that they result from the extended presentation time in the JOL task ( $M=4.3 \mathrm{~s}$ ) relative to the read task (fixed at $2 \mathrm{~s}$ ). However, a more meaningful interpretation is that JOLs enhanced the processing of differences among the homogeneous background items. This enhanced processing led to improved recall of items that would otherwise be very similar to one another, such as a group of words from a single category or a homogeneous group of two-digit numbers. This interpretation is consistent with the finding that JOLs reduced the isolation effect.

\section{General discussion}

People often remember things that are different, unusual, or distinctive. However, we sometimes fail to notice things that are different or we quickly forget different items that we noticed. The challenge for memory researchers is to determine the conditions under which we will and will not observe the positive effects of distinctiveness on memory.

Items isolated in the middle of a series are better remembered than control items using a wide variety of dimensional contrasts, materials, and tasks (see Schmidt, 1991, 2008, 2012; and Wallace, 1965; for reviews). Researchers have offered two common classes of explanations for these findings. First, they have suggested that isolated items receive increased processing at input (e.g., Green, 1956; Rundus, 1971; Schmidt, 1991). Second, they have argued that distinctive items are easier to retrieve or discriminate from background items than non-distinctive items (e.g., Kelley \& Nairne, 2001; Waddill \& McDaniel, 1998). Repeated demonstrations of the early isolation effect challenged the idea that extra processing is necessary to support good memory for distinctive events (e.g., Hunt, 1995). The research presented above was designed to explore the generality of the early isolation effect, and thus revisit the role of attention and extra processing in supporting good memory for distinctive events.

Experiment 1a provided a direct comparison between the midlist and early isolation effects. Target items were isolated by conceptual category, and memory for isolated items was compared to memory for the same items in either homogeneous lists of categorized words or lists of unrelated words. Midlist isolates were remembered better than targets in both comparison lists. In contrast, targets isolated early in the list were not remembered any better than targets in unrelated lists. More interestingly, early isolates were remembered more poorly than comparison items that were congruent with the background conceptual category. This pattern of early isolation effects with unrelated and homogeneous comparisons lists was replicated in Experiment $1 \mathrm{~b}$ and extended to a nondistracting read condition.

Under what conditions will an early isolation effect be observed? Experiment 2 was designed to answer this question by isolating words by conceptual category, isolating words in lists of numbers, and isolating numbers in lists of words from a shared category. The early isolation effect was only observed when words and numbers were isolated in lists of numbers and words, respectively. Simple conceptual differences between isolates and background items are not sufficient to produce an early isolation effect. To observe the early isolation effect, researchers must employ differences that are easily or automatically encoded such as words versus numbers, or they must use processing tasks that highlight target/background contrasts such as the delayed JOLs employed by Geraci and Manzano (2010) and Smith and Hunt (2016). Smith and Hunt (2016) argued that delayed JOLs enhance processing of categorical information, thereby increasing the likelihood of a conceptual isolation effect. The only reported exception to this rule is the Smith (2011, Exp. 1) article. $^{6}$

We have suggested that participants code the differences between numbers and words automatically. Word/number differences may also support the enhanced retrieval and discrimination processes that underlie the isolation effect. However, even the word/number isolation effect for early isolates was somewhat fragile in Experiment 2. Word/number contrasts did not produce an early isolation effect when homogeneous comparison lists were used and participants performed a JOL task encouraging them to process the differences among ostensibly similar background items in the lists. This result should remind us of the Hunt and Lamb (2001) finding that difference

\footnotetext{
${ }^{6}$ Differences between the Smith (2011) study and the experiments reported above include list length (eight words in the Smith study vs. 15 words in the present experiments), number of lists presented (1 vs. 6), duration of item presentation ( 3 vs. $2 \mathrm{~s}$ ), and timing of the recall test (delayed vs. immediate). Any or all of these variables could have produced the discrepant results.
} 
judgments reduced the midlist isolation effect when words were isolated in contrasting conceptual categories.

The results of Experiments 1a, 1b, and 2 are consistent with the three-factor framework for understanding distinctiveness developed in Schmidt (2012) and further elaborated upon in Schmidt and Schmidt (2015). The first factor in this framework suggests that certain emotional or arousing stimuli automatically attract attention and encoding resources, and thus are well remembered in most contexts. Such stimuli include nudes (Schmidt, 2002), spiders (Schmidt, 2007), and taboo words (Schmidt \& Saari, 2007; Schmidt \& Schmidt, 2015). The second factor emphasizes the roles of context and task demands in the selection and weighting of features for storage in memory. For example, participants may code font color when they encounter a few red-font words in a list of words printed primarily in black font, even though the color dimension is typically irrelevant to the task of reading. Similarly, participants may give high weight to category information when they encounter a word from a contrasting category in a list of words from a common conceptual category. The third factor in the Schmidt framework considers the usefulness of the sampled features for the memory task. Knowing the category shared by all the words in a single-category list or by the background words in a conceptual isolation list can greatly aid retrieval. In contrast, category information is not very useful in recalling a list of unrelated words. The three factors in the Schmidt framework can combine in interesting ways. For example, recalling a taboo word from a list of neutral words is aided both by increased attention to the taboo word and the ease of retrieving a single taboo word from an otherwise homogeneous list of unemotional items.

Schmidt and Schmidt's (2015) contribution to this framework was clarifying the role of attentional resources in the encoding process. Participants are unlikely to code category changes in a list when their attention is directed to another, irrelevant, stimulus dimension. Their failure to notice categorical information can lead to poor memory for items that are conceptually distinctive. However, attention is not an all-ornone process. Some stimulus contrasts may be coded relatively easily or even automatically. Bireta and Mazzei (2015) reported that midlist isolation by conceptual category did not enhance recall when participants were performing a distracting task at encoding. However, they observed the physical isolation effect using contrasts in font color between the isolates and background words under distracted conditions at encoding. Our interpretation of the Bireta and Mazzei results is that noting and coding the conceptual category of words requires greater cognitive resources than noting and coding physical changes such as font color.

When we apply the Schmidt framework to the early isolation effect, we can assume that participants must notice and store in memory the relevant stimulus change in order for the effect to occur. Further, the change that is noticed and encoded must be useful on the memory test. We already know from Schmidt and Schmidt (2015) that a change from one conceptual category to another is not automatically coded. In fact, detecting conceptual changes appears to require considerable attentional resources. When words are isolated early in a series (before list structure is apparent), participants may fail to notice conceptual contrasts unless specifically directed to by the task (as in the delayed JOL and immediate category verification tasks of Geraci \& Manzano, 2010). However, Experiment 2 suggests that the difference between a twodigit number and a word is automatically encoded. Furthermore, participants can use the information that a list contained one number and the names of different fish to guide and aid their recall. Note that we are not arguing that the isolation effect is simply the result of increased attention to isolated items as suggested by early researchers (e.g., Green, 1956). Rather, the isolation effect results from changes in how resources are allocated during study, the type of processing that target and background items receive, the relevance of that processing to the isolation contrast, the structure of the memory set, and the retrieval advantages enjoyed by isolated items. In fact, the results of Experiment 2 clearly demonstrated an isolation effect with the digit/word contrast when it was unlikely that the early isolates received extra attention. Thus, extra attention is not a necessary cause of the isolation effect.

In summary, the early isolation effect has been studied since von Restorff's original article outlining the effects of stimulus contrasts on memory performance. Popular theoretical explanations of the isolation effect assume that the effect is fairly general and is independent of the serial position of the isolated item. The research presented above demonstrated that the early isolation effect does not occur under conditions that do support the midlist isolation effect. Thus, the early isolation effect may be of more limited generality than many researchers have assumed. The three experiments presented here add to a growing literature demonstrating the importance of the type of stimulus contrast, the participant's allocation of attentional resources, and the retrieval advantages of comparison lists in supporting memory for distinctive events in the isolation paradigm.

Author note The authors thank our students who contributed to the conduct of this research, including Nathaniel W. Coggins, Sommer A. Franklin, Cuauhtemoc U. Gonzalez, Brandon L. Goode, Jared D. Gunther, Jessica T. Howell, Melissa C. Millikan, Carl B. Nabors, Tabatha R. Slate, Ashley L. Stewart, and Tamara A. Tucker.

\section{References}

Bellezza, F. S., \& Cheney, T. L. (1973). Isolation effect in immediate and delayed recall. Journal of Experimental Psychology, 99, 55-60. doi: $10.1037 / \mathrm{h} 0034756$ 
Bireta, T. J., \& Mazzei, C. M. (2015). Does the isolation effect require attention? Memory \& Cognition, 44, 1-14. doi:10.3758/s13421015-0538-y

Bireta, T. J., Surprenant, A. M., \& Neath, I. (2008). Age-related differences in the von Restorff isolation effect. Quarterly Journal of Experimental Psychology, 61, 345-352. doi:10.1080 /17470210701626608

Bone, R. N., \& Goulet, L. R. (1968). Serial position and the von Restorff isolation effect. Journal of Experimental Psychology, 76, 494-496. doi: $10.1037 / \mathrm{h} 0025505$

Cimbalo, R. S., \& Brink, L. (1982). Aging and the von Restorff isolation effect in short/term memory. Journal of General Psychology, 106, 69-76. doi:10.1080/00221309.1982.9710974

Dunlosky, J., Hunt, R., \& Clark, E. (2000). Is perceptual salience needed in explanations of the isolation effect? Journal of Experimental Psychology: Learning, Memory, and Cognition, 26, 649-657. doi:10.1037/0278-7393.26.3.649

Elhalal, A., Davelaar, E. J., \& Usher, M. (2014). The role of the frontal cortex in memory: An investigation of the von Restorff effect. Frontiers in Human Neuroscience, 8, 410. doi:10.3389 /fnhum.2014.00410

Fabiani, M. (2006). Multiple electrophysiological indices of distinctiveness. In R. R. Hunt \& J. B. Worthen (Eds.), Distinctiveness and memory (pp. 339-360). New York, NY: Oxford University Press. doi:10.1093/acprof:oso/9780195169669.003.0015

Fabiani, M., \& Donchin, E. (1995). Encoding processes and memory organization: A model of the von Restorff effect. Journal of Experimental Psychology: Learning, Memory, and Cognition, 21, 224-240.

Fabiani, M., Karis, D., \& Donchin, E. (1990). Effects of mnemonic strategy manipulation in a Von Restorff paradigm. Electroencephalography \& Clinical Neurophysiology, 75, 22-35. doi:10.1016/0013-4694(90)90149-E

Gati, I., \& Ben-Shakhar, G. (1990). Novelty and significance in orientation and habituation: A feature-matching approach. Journal of Experimental Psychology: General, 119, 251-263. doi:10.1037 /0096-3445.119.3.251

Geraci, L., \& Manzano, I. (2010). Distinctive items are salient during encoding: Delayed judgements of learning predict the isolation effect. Quarterly Journal of Experimental Psychology, 63, 50-64. doi:10.1080/17470210902790161

Geraci, L., McDaniel, M. A., Manzano, I., \& Roediger, H. L., III. (2009). The influence of age on memory for distinctive events. Memory \& Cognition, 37, 175-180. doi:10.3758/MC.37.2.175

Green, R. T. (1956). Surprise as a factor in the von Restorff effect. Journal of Experimental Psychology, 52, 340-344. doi:10.1037/h0047496

Howe, M. L., Courage, M. L., Vernescu, R., \& Hunt, M. (2000). Distinctiveness effects in children's long-term retention. Developmental Psychology, 36, 778-792. doi:10.1037/00121649.36.6.778

Huang, I. N., Scale, J., \& McIntyre, R. (1976). The von Restorff isolation effect in response and serial order learning. Journal of General Psychology, 94, 153-165.

Huang, I. N., \& Wille, C. (1979). The von Restorff isolation effect in free recall. Journal of General Psychology, 101, 27-34.

Hunt, R. R. (1995). The subtlety of distinctiveness: What von Restorff really did. Psychonomic Bulletin \& Review, 2, 105-112. doi:10.3758 /BF03214414

Hunt, R. R. (2009). Does salience facilitate longer-term retention? Memory, 17, 49-53. doi:10.1080/09658210802524257

Hunt, R. R., \& Lamb, C. A. (2001). What causes the isolation effect? Journal of Experimental Psychology: Learning, Memory, and Cognition, 27, 1359-1366. doi:10.1037/0278-7393.27.6.1359

Ischebeck, A., Heim, S., Siedentopf, C., Zamarian, L., Schocke, M., Kremser, C., ... Delazer, M. (2008). Are numbers special? Comparing the generation of verbal materials from ordered categories (months) to numbers and other categories (animals) in an fMRI study. Human Brain Mapping, 29, 894-909. doi:10.1002 /hbm.20433

Janschewitz, K. (2008). Taboo, emotionally valenced, and emotionally neutral word norms. Behavior Research Methods, 40, 1065-1074. doi:10.3758/BRM.40.4.1065

Johansson, B. S. (1970). Attention and the von Restorff effect. British Journal of Psychology, 61, 163-170.

Karis, D., Fabiani, M., \& Donchin, E. (1984). "P300” and memory: Individual differences in the von Restorff effect. Cognitive Psychology, 16, 177-216. doi:10.1016/0010-0285(84)90007-0

Kelley, M. R., \& Nairne, J. S. (2001). von Restorff revisited: Isolation, generation, and memory for order. Journal of Experimental Psychology: Learning, Memory, and Cognition, 27, 54-66. doi:10.1037/0278-7393.27.1.54

Koriat, A. (1997). Monitoring one's own knowledge during study: A cueutilization approach to judgments of learning. Journal of Experimental Psychology: General, 126, 349-370. doi:10.1037 /0096-3445.126.4.349

Ludwig, B. P. (2005). The incongruity hypothesis and the early isolation effect: Comparing the role of primary and secondary distinctive targets in the early isolation effect (unpublished doctoral dissertation), Texas A\&M University, Commerce, TX.

Malhotra, A., \& Dixit, R. C. (1982). The von Restorff effect as a function of the conditions of recall and the serial position of the item. Archivio di Psicologia, Neurologia e Psichiatria, 43, 378-389.

McConnell, A. R., Sherman, S. J., \& Hamilton, D. L. (1994). Illusory correlation in the perception of groups: An extension of the distinctiveness-based account. Journal of Personality and Social Psychology, 67, 414-429. doi:10.1037/0022-3514.67.3.414

Otani, H., Von Glahn, N. R., Libkuman, T. M., Goernert, P. N., \& Kato, K. (2014). Emotional salience and the isolation effect. Journal of General Psychology, 141,35-46. doi:10.1080 /00221309.2013.848180

Pillsbury, W. B., \& Raush, H. L. (1943). An extension of the KöhlerRestorff inhibition phenomenon. American Journal of Psychology, 56, 293-298. doi:10.2307/1417511

Postman, L., \& Phillips, L. W. (1954). Studies in incidental learning: I. The effects of crowding and isolation. Journal of Experimental Psychology, 48, 48-56. doi:10.1037/h0060802

Rundus, D. (1971). Analysis of rehearsal processes in free recall. Journal of Experimental Psychology, 89, 63-77. doi:10.1037/h0031185

Saltzman, I. J., \& Carterette, T. S. (1959). Incidental and intentional learning of isolated and crowded items. The American Journal of Psychology, 72, 230-235.

Schmidt, S. R. (1991). Can we have a distinctive theory of memory? Memory \& Cognition, 19, 523-542. doi:10.3758/BF03197149

Schmidt, S. R. (2002). Outstanding memories: The positive and negative effects of nudes on memory. Journal of Experimental Psychology: Learning, Memory, and Cognition, 28, 353-361. doi:10.1037/02787393.28.2.353

Schmidt, S. R. (2006). Emotion, significance, distinctiveness, and memory. In R. R. Hunt \& J. B. Worthen (Eds.), Distinctiveness and memory (pp. 47-64). New York, NY: Oxford University Press. doi:10.1093/acprof:oso/9780195169669.003.0003

Schmidt, S. R. (2007). Unscrambling the effects of emotion and distinctiveness on memory. In J. S. Nairne (Ed.), The foundations of remembering: Essays in honor of Henry L. Roediger III (pp. 141158). New York, NY: Psychology Press.

Schmidt, S. R. (2008). Distinctiveness and memory: A theoretical and empirical review. In J. Byrne \& H. L. Roediger III (Eds.), Learning and memory: A comprehensive reference. Vol. 2: Cognitive psychology of memory (pp. 125-144). Oxford, UK: Elsevier.

Schmidt, S. R. (2012). Extraordinary memories for exceptional events. New York, NY: Psychology Press. 
Schmidt, S. R., \& Saari, B. (2007). The emotional memory effect: Differential processing or item distinctiveness? Memory \& Cognition, 35, 1905-1916. doi:10.3758/BF03192924

Schmidt, S. R., \& Schmidt, C. R. (2015). Inattentional blindness and the von Restorff effect. Memory \& Cognition, 43, 151-163. doi:10.3758/s13421-014-0459-1

Smith, R. E. (2011). Providing support for distinctive processing: The isolation effect in young and older adults. Psychology and Aging, 26, 744-751. doi:10.1037/a0022715

Smith, R. E., \& Hunt, R. R. (2016). Distinctive processing in young and older adults: Metacognitive judgments and the isolation paradigm. Psychology and Aging, 31, 198. doi:10.1037/pag0000076

Soderstrom, N. C., Clark, C. T., Halamish, V., \& Bjork, E. L. (2015). Judgments of learning as memory modifiers. Journal of Experimental Psychology: Learning, Memory, and Cognition, 41, 553-558. doi:10.1037/a0038388

Tulving, E. (1969). Retrograde amnesia in free recall. Science, 164, 8890. doi:10.1126/science.164.3875.88
Van Overschelde, J. P., Rawson, K. A., \& Dunlosky, J. (2004). Category norms: An updated and expanded version of the Battig and Montague (1969) norms. Journal of Memory and Language, 50, 289-335. doi:10.1016/j.jml.2003.10.003

Vitali, P., Minati, L., Chiarenza, G., Brugnolo, A., Girtler, N., Nobili, F., ... Rodriguez, G. (2006). The Von Restorff effect in ageing and Alzheimer's disease. Neurological Sciences, 27, 166-172. doi:10.1007/s10072-006-0662-3

von Restorff, H. (1933). Über die Wirkung von Bereichsbildungen im Spurenfeld [On the effect of field formations in the trace field]. Psychologische Forschung, 18, 299-342. doi:10.1007/BF02409636

Waddill, P. J., \& McDaniel, M. A. (1998). Distinctiveness effects in recall. Memory \& Cognition, 26, 108-120. doi:10.3758/BF03211374

Wallace, W. P. (1965). Review of the historical, empirical, and theoretical status of the von Restorff phenomenon. Psychological Bulletin, 63, 410-424. doi:10.1037/h0022001 